Abstract ID: 79

\title{
Bilateral total knee replacement in severe knee osteoarthritis with large bone defect using autologous bone graft augmentation
}

Mohd Shahidan Noor Rahin | Khairul Nizam Siron | Ed Simor Khan Mor Japar Khan | Ahmad Hafiz Zulkifly I Nik Fatmy Nik Najmy

Department of Orthopaedics, Traumatology \& Rehabilitation, Kulliyyah of Medicine, International Islamic University Malaysia

40 years old malay gentleman with underlying gout presenting with severe pain in both knees for 10 years, analgesic dependant and wheelchair ambulation. Examination of right knee shows fixed flexion at 10 degrees, range of movement 10-100 degree flexion, lateral collateral laxity and varus deformity. Left knee had fixed flexion at 10 degrees, range of movement 5-100 degree and varus deformity. Blood investigation showed uric acid. X-ray of bilateral knee showed severe tricompartmental arthritis with large medial tibial defect. The patient has undergone bilateral knee replacement in 5 months interval started with left knee. Surgical technique is the same for both knees, intraoperative shows a large posteromedial bone defect. We used $5 \mathrm{~mm}$ metal augmentation on the right knee, but due to limited financial resources, we have to use the bone graft from the osteotomized bone for the augmentation on the left side. The bone augmentation was fixed with cancellous screw. The patient was referred to physiotherapy for range of motion exercises. 1 year post operatively patient able to ambulate without aid. Pain score improved. The patient does not require analgesia. Functional and knee score is excellent. Range of movement, bilateral knee are 0-100 degree. Radiological finding shows no evidence of construct failure in both metal block and bone graft construct. Severe arthritis with bone defect is common due to late presentation. Using autologous bone graft can provide good result as using expensive meta block for reconstruction.

KEYWORDS: severe arthritis, posteromedial bone defect 